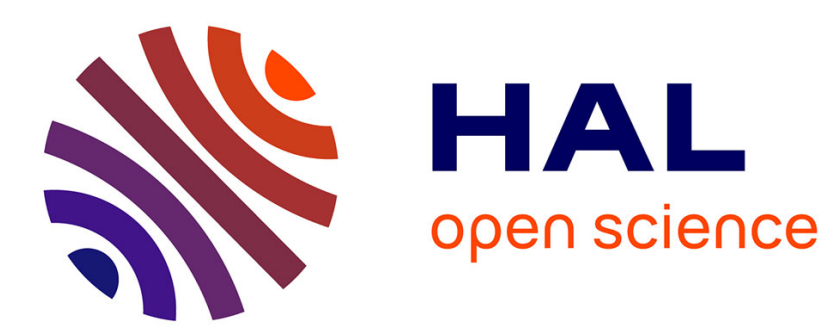

\title{
RECENT ASPECTS OF THE NUCLEAR SPIN ISOSPIN RESPONSE FUNCTION
}

\author{
M. Ericson
}

\section{To cite this version:}

M. Ericson. RECENT ASPECTS OF THE NUCLEAR SPIN ISOSPIN RESPONSE FUNCTION. International Symposium On Highly Excited States And Nuclear Structure ,Hesans 83, 1983, Orsay, France. pp.C4-489-C4-501, 10.1051/jphyscol:1984439 . jpa-00224105

\section{HAL Id: jpa-00224105 https://hal.science/jpa-00224105}

Submitted on 1 Jan 1984

HAL is a multi-disciplinary open access archive for the deposit and dissemination of scientific research documents, whether they are published or not. The documents may come from teaching and research institutions in France or abroad, or from public or private research centers.
L'archive ouverte pluridisciplinaire HAL, est destinée au dépôt et à la diffusion de documents scientifiques de niveau recherche, publiés ou non, émanant des établissements d'enseignement et de recherche français ou étrangers, des laboratoires publics ou privés. 


\section{RECENT ASPECTS OF THE NUCLEAR SPIN ISOSPIN RESPONSE FUNCTION}

\section{Ericson}

Institut de Physique Nucléaire (et IN2P3), Université Claude Bermard, Lyon I, 43, boulevard du 11 Novembre 1918, 69622 Vilieurbanne Cedex, France

Résumé - Divers aspects de la réponse nucléaire de spin isospin sont discutés, en particulier les aspects collectifs de la réponse lplh ainsi que la réponse due aux excitations $2 \mathrm{p} 2 \mathrm{~h}$.

Abstract - Various aspects of the nuclear response to spin isospin excitations are discussed, in particular the collective features of the lplh response and the response arising from the $2 \mathrm{p} 2 \mathrm{~h}$ excitations.

The subject of my talk is the response fuction of the nucleus to a spin isospin excitation. I will focus on some recent aspects, namely the collective features of the lplh response in the region of the quasi elastic peak and the response due to $2 \mathrm{p} 2 \mathrm{~h}$ excitations. As an application of these problems I will finaliy mention the so-called EMc effect furopean muon collaboration).

What is the interest of these problems; what are the open issues ? The question of collective effects in the region of the quasi elastic peak is an interesting subject in itself, but particularly in the spin isospin channel because it is intimately linked to the pion exchange force. It will thus shed light in the existence and the role of the pion in nuclei which is sometimes questioned nowadays and for which a convincing evidence should be built. The question of the $2 \mathrm{p} 2 \mathrm{~h}$ response is directly related to the problem of the high energy tail of the Gamow-Teller (G.T) strength fuction; we will also discuss the problem of its quenching. Finaly the EMC effect in its pionic interpretation offers a beautiful application of these ideas in a very high energy experiment.

In order to fix the notations let me remind you the definition of the response function. I will adopt throughout this work an infinite nuclear matter description for which it is convenient to work in momentum space. If an external force $U(q, \omega)$ is applied to the nuclear system at a momentum $q$ and frequency w then a density fluctuation on is induced

$$
\delta_{n}(q, \omega)=\pi(q, \omega) \quad U(q, \omega)
$$

The quantity $\pi$ is the polarization propagator. Its imaginary part is the response function of the system. It is a quantity of a great practical interest since it is proportional to the inelastic cross section for a probe which couples to these density fluctuations. It is also (within trivial factors) the correlation function of the system.

The response of the system is linked to its excitations. For a free Fermi gas the nucleons respond individually and the excitations are of two types, the purely nuclear ones at low energies, $\omega \approx q^{2} / 2 m_{N}$ and the 
nucleonic ones at high energy, for a spin isospin excitation mostly due to the $\Delta, \omega \approx \omega_{\Delta}+q^{2} / 2 \mathrm{~m} \Delta$ with $\omega_{\Delta}=300 \mathrm{MeV}$.

For a fixed value of $q$ the behavior of $\pi$ is as follows. There are two regions of response, one at low energy and one at high energy, as is shown in $\mathrm{Fig} . \mathrm{l}$. Re $\pi$ has a dispersive behavior, Re ${ }^{N}$ changing sign at low energy while Re $\pi^{\circ}$ changes sign at high energy. I would like to draw your attention to the fact that $\operatorname{Re} \pi^{N}$ (and hence Re $\pi^{\circ}$ ) is not, at fixed $q$, a smooth function of $\omega$ in the low energy sector or equivalently, at fixed $w$, it is not a smooth function of $q$. Moreover there is a near cancellation between $R e \pi^{N}$ and $R e \pi^{0}$ in a certain kinematical domain (see Fig.l).

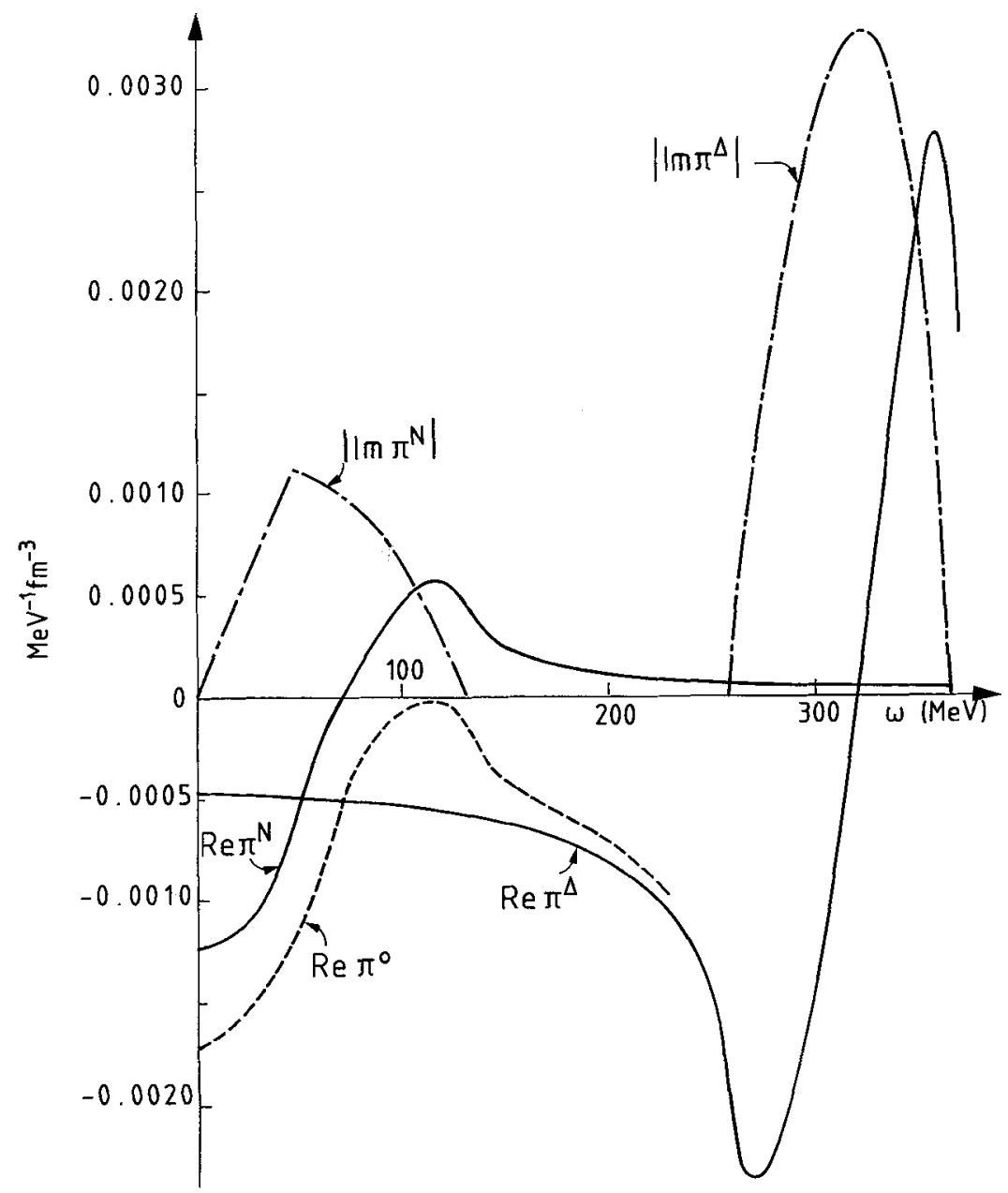

Fig. 1 - Imaginary parts (dot-dashed lines) and Real parts of $\pi^{N}$ and $\pi^{\circ}$ (continuous lines) and of $\pi^{\circ}=\pi^{N}+\pi^{\Delta}$ (dashed line). 
When the interaction between the nucleons is switched on the nucleons no longer respond individually. The external force can be transmitted to the neighbours through a coherent superposition of lplh states and the lplh response becomes

$$
\pi(q, w)=\frac{\pi^{0}(q, w)}{1-V(q, \omega) \pi^{0}(q, w)}
$$

where $\mathrm{V}$ is the ph. interaction. This expression sums the following series of bubble graphs.

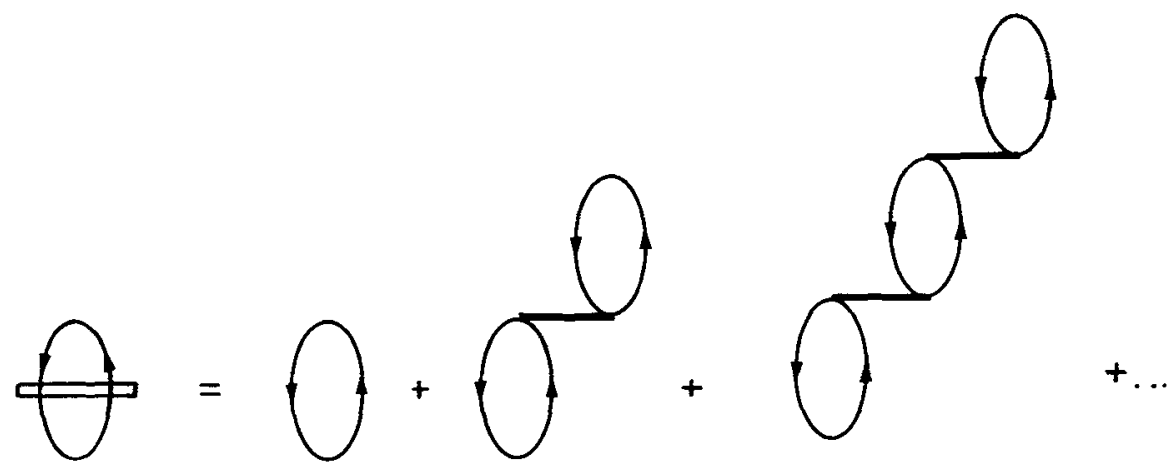

The expression (I) represents the random phase approximation, RFA, more exactly a simplified version of it where the antisymmetrisation is omitted.

Although oversimplified as compared to the exact RPA, expression (1) is a useful guideline to study the collective features of the response, embodied in the RPA denominator, and to predict what kind of features should be expected.

This approximation gives for the response

$$
\operatorname{Im} \pi(q, \omega)=\frac{\operatorname{Im} \pi^{0}(q, \omega)}{\left|1-V(q, \omega) \operatorname{Re} \pi^{0}(q, \omega)\right|^{2}+\left|V(q, \omega) \operatorname{Im} \pi^{0}(q, \omega)\right|^{2}}
$$

The collective effects influence the response in a way which depends on the sign of the product $V(q, \omega) \operatorname{Re} \pi^{0}(q, \omega)$.

For what concerns the p.h. Force the pion which couples to the nucleonic spin and isospin is an effective agent to carry the spin excitation. This makes one of the interesting aspects of the field. However the pion has $a \vec{\sigma} \cdot \vec{q}$ type of coupling. Consequently it can act only (in nu- 
clear matterl if the exciting field itself has a similar coupling,i.e., if it probes the component of the magnetization along the direction of the momentum. If the probe couples in a transverse way $(\vec{\sigma} \times \hat{q})$ then in nuclear matter it is totally blind to the pion exchange force, at least for the direct piece but remember that we ignore antisymmetrisation in our simple approximation.

This particular feature turns out to be of great value. By turning the difection of the magnetization that we explore we can switch on and off: the pionic component of the force, enabling thus the display of this component. In practice this is not so easy to carry through but the possibility is there and it should be exploited. What it means in practice is that the force and hence the collective features that this force generates are different in the spin longitudinal and in the spin transverse channels (1).

Beside pion exchange the force contains short-range components which are parametrized phenomenologically by the Landau-Migdal parameter g', which represents the repulsive short range component. The total force $V_{L}$ in the spin longitudinal channel is thus

$$
V_{L}=\frac{f^{2}}{m_{\pi}^{2}} \quad\left[g^{\prime}-\frac{q^{2}}{q^{2}+m_{\pi}^{2}-\omega^{2}}\right]
$$

Where $f$ is the $\pi N$ coupling constant $\left(f^{2} \approx 1\right)$. The pion exchange piece generates a rapid variation in momentum due to the small value of the pion mass.

In the spin transverse channel there is no pion exchange and the force $V_{\text {T }}$ has a slow variation in $q$ (maybe due to p exchange) remaining repulsive over a larger range of momenta. The two forces are represented in Fig. 2 for a typical value $g^{\prime}=0.7$.

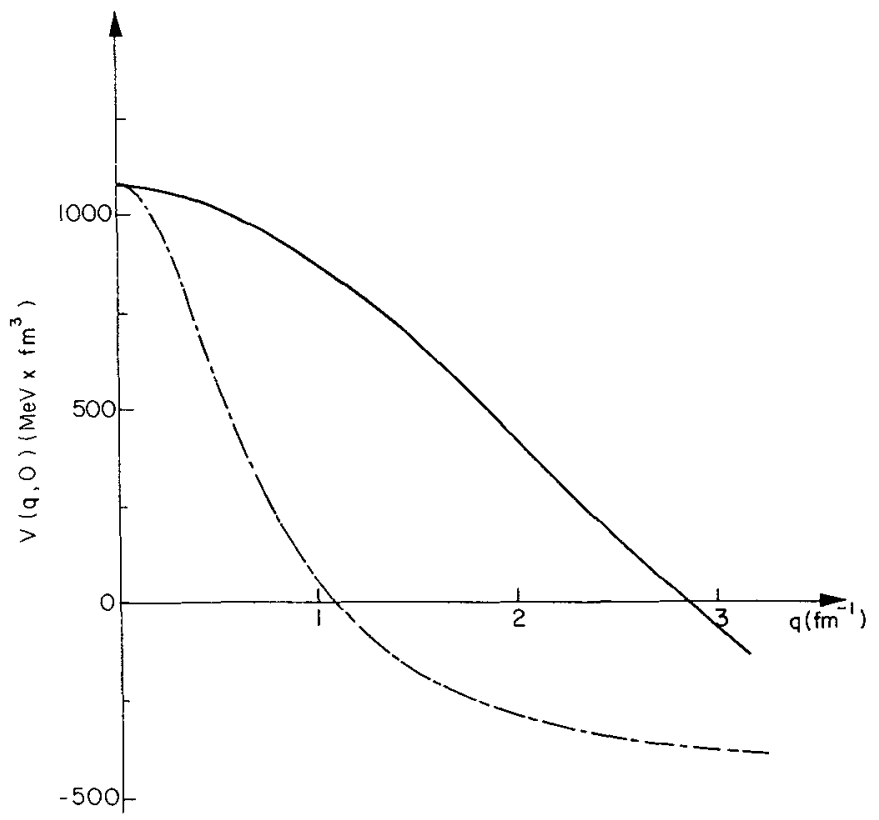

Fig. 2 - The p.h. force in the longitudinal (dash-dotted line) and in the transverse channel (continuous line) for $\mathrm{g}^{\prime}=0.7$. 
Beyond $q \approx 1 \mathrm{fm}^{-1}$ the two forces differ violently; one is mildy attractive while the other is appreciably repulsive. The collective features of the two responses, which reflect the nature of the force, are then expected to differ markedly. This is illustrated in Fig. 3 which represents the responses as a function of $\omega$ at a fixed momentum $\mathrm{q}=1.3 \mathrm{fm}^{-1}$. The free Fermi gas value is shown together with the responses obtained in the RPA expression (2) both in the longitudinal and in the spin transverse channel. The contrast is clearly visible : the transverse response is quenched and hardened (its maximum is displaced at larger $\omega$ values) while the longitudinal one is enhanced and softened.

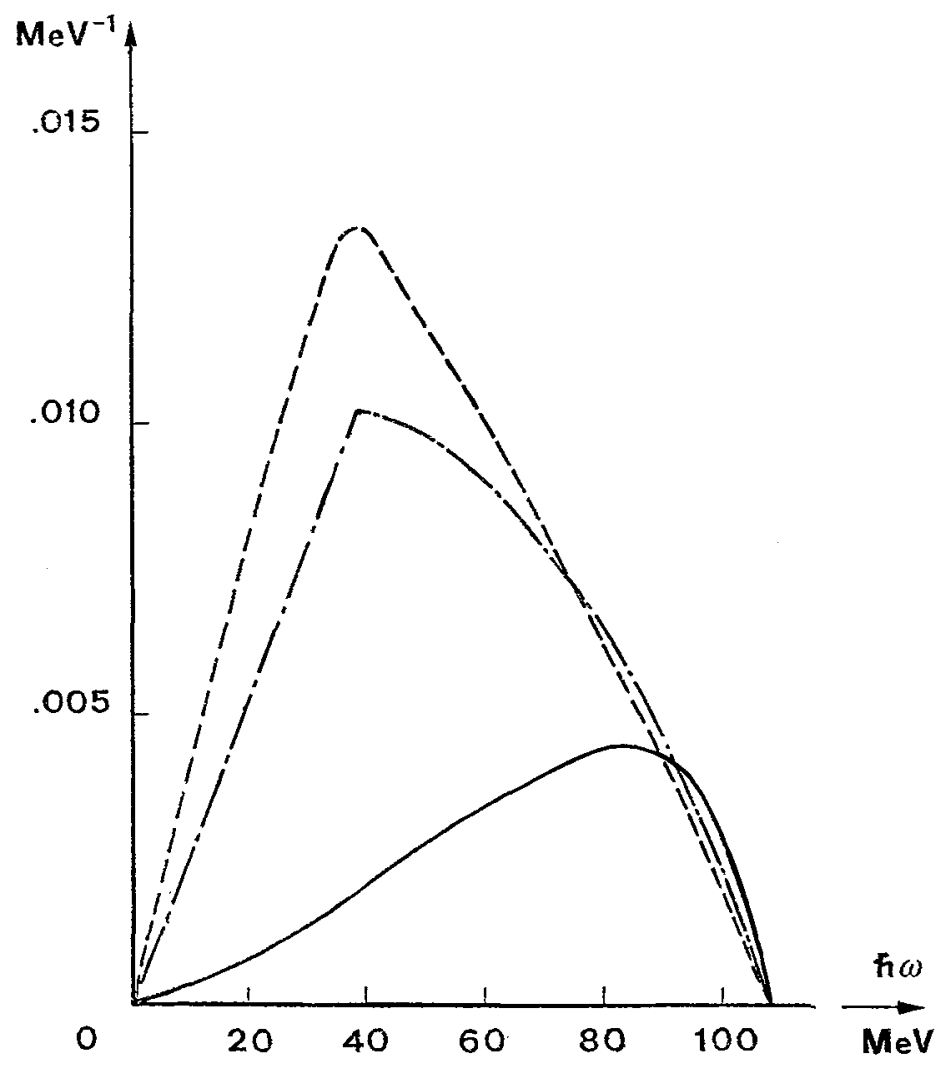

Fig. 3 - The response function at $q=1.3 \mathrm{fm}^{-1}$ in the free case (dashdotted line) and in the RPA approximation for the longitudinal (dotted line) and transverse (continuous line) channel.

The hardening of the transverse response is a remnance of the collective state, the zero sound, which exists at low momentum ( $q \leqslant 0.4 \mathrm{fm}$ ). At larger $q$ the collective state has evolved and entered the p.h. continuum where it survives in the form of a hardening of the response.

The softening of the longitudinal response is also the reflect of another collective state, the pion condensate, which would occur at $\omega=0$ and a critical momentum $q_{\approx} \approx 2 \mathrm{fm}^{-1}$. However this state does not occur at the ordinary density but maybe at a much larger one $\left(\rho>3 \rho_{0}\right)$ and therefore the softening effect is not very pronounced. In addi- 
tion the momentum is not the best to display it, the effect would be more pronounced at the critical momentum.

The interesting feature of the contrast is that it is practically independant of $\mathrm{g}^{\prime}$. If $\mathrm{g}^{\prime}$ is increased the softening and enhancement of the longitudinal response fade away but the quenching and hardening of the transverse one become more pronounced, in such a way that the contrast remains intact. It is entirely due to pion exchange, therefore its display would sign unambiguously the pion exchange force in a region of a space like momentum transfer. It would also indicate the collective nature of at least one of the two responses, longitudinal or transverse.

At present no data exist on the spin longitudinal response, the reason being that few probes have a $\sigma . \hat{q}$ type of coupling. We are awaiting for the results of the Los Alamos experiment ${ }^{2}$ on the polarization transfer in inclusive $\mathrm{pp}^{\prime}$ reactions on nuclei. It will provide the two responses, which eliminates a number of systematic uncertainties in the comparison.

In the meantime we can investigate the situation for the transverse response where we have at our disposal an ideal probe, ee' scattering. The separation between the transverse and the longitudinal (charge) responses has been performed in a number of cases. The transverse one is essentially magnetic; it is also isovector since the isovector magnetic moment of the nuclifon largely dominates over the isoscalar one. An example of the data is shown in Fig.4 in the case of $56 \mathrm{Fe}$ for a fixed momentum $q=1,65 \mathrm{fm}^{-1}$. Also shown is the lplh cross section obtained for a free Fermi gas and the one obtained in the simplified RPA expression (2) with $g^{\prime}=0.7$. Both description totally fail in the large energy region but this is to be expected since the $2 \mathrm{p} 2 \mathrm{~h}$. excitations which take over in that energy range are ignored in this description. We will come back to that question.

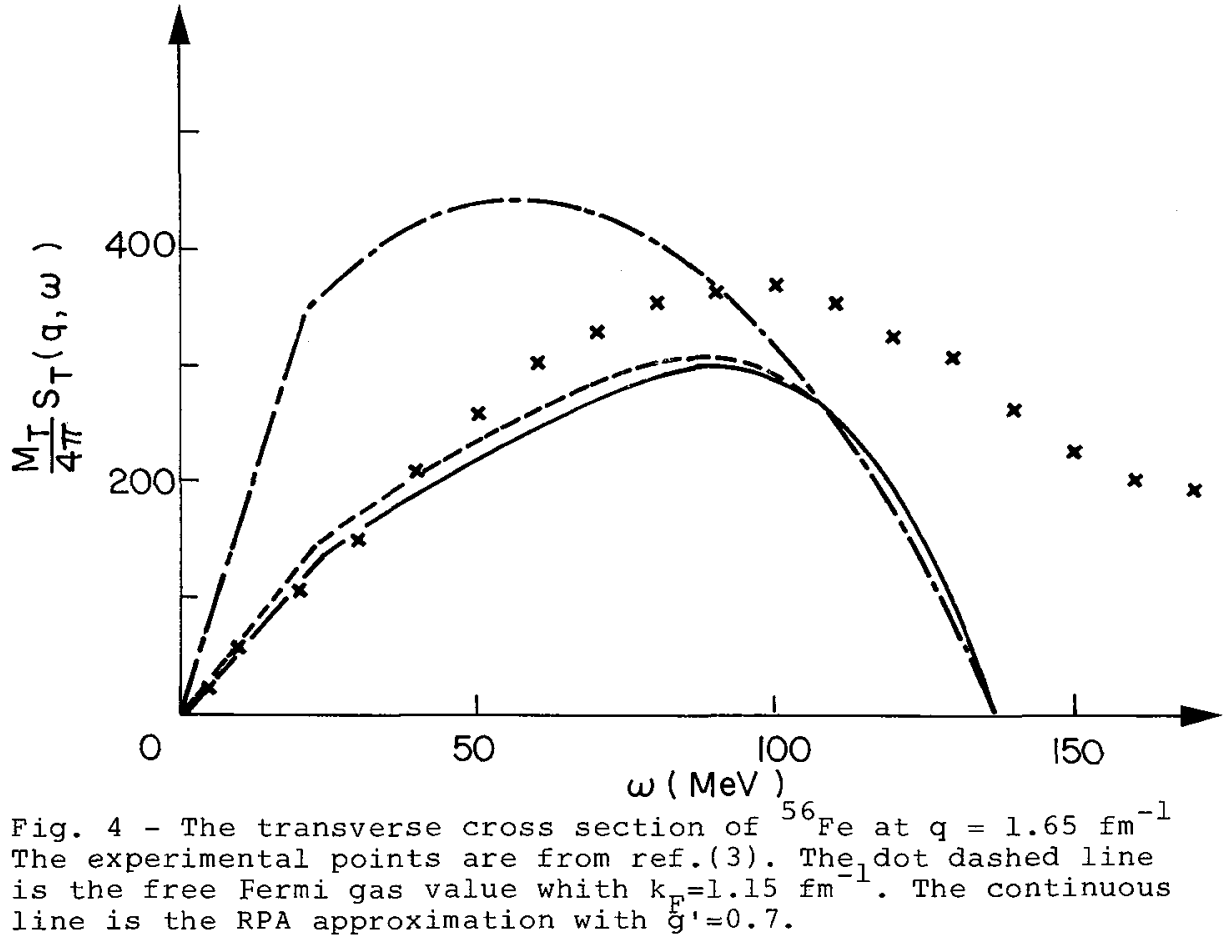


In the low energy region instead the RPA expression seems to do rather well, indicating the collective character of the response. However this evidence is not conclusive since the description which ignores HartreeFock effects, antisymrnetrisation etc... is too rough. In order to display the existence of collectivity in one single response a much more refined_description is, needed. An encouraging point in that respect is that an analysis (4) of the recent Saclay data has shown that once the trivial factors are eliminated in the cross section the response becomes universal for ${ }^{40} \mathrm{Ca},{ }^{48} \mathrm{Ca}$ and ${ }^{56} \mathrm{Fe}$. This shows that the details of the shell structure are no longer relevant at these momenta and that one indeed probes the bulk properties of nuclear matter.

I come now to the high energy region where the $2 \mathrm{p} 2 \mathrm{~h}$ excitations dominate, as was shown by several studies, either microscopic or phenomenological. I will describe here the recent approach of Alberico et al. (5) It has both a microscopic and a phenomenological aspect. The microscopic description amounts to incorporating all the graphs which are responsible for the absorption of the photon in the framework of a perturbative theory. They are of four kinds.

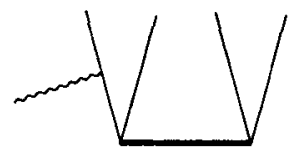

a

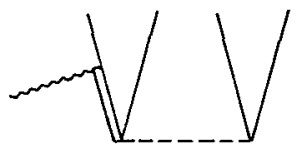

b

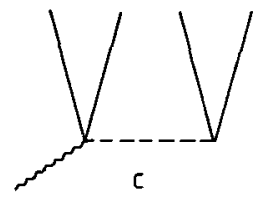

c

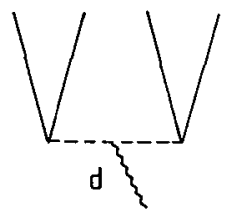

Fig. 5 - Graphs responsible for the photon absorption.

The first one represents the absorption by a correlated pair of nucleons. In the second one the $\gamma$ excites virtually a $\Delta$. The third and fourth ones are graphs of the meson exchange type, the $\gamma$ photoproducing a pion via a contact interaction or being absorbed by a pion in flight. The interference between all these terms is incorporateci.

For the NN interaction which builds the correlations we keep only the spin isospin component of the ph force and we take it to be consistent with a G matrix evaluation by Dickoff (6).

The result of this lengthy calculation is shown in Fig. 6 which displays the ee' transverse cross section for $q=1.85 \mathrm{fm}^{-1}$. The $2 \mathrm{p} 2 \mathrm{~h}$ contribution is shown, as well as the total result which incorporates also the lplh contribution evaluated in our previous RPA scheme. The incorporation of the $2 \mathrm{p} 2 \mathrm{~h}$ part improves remarkably the fit, in particular in the region between the nuclear and $\Delta$ sector. The agreement turns out even better than expected on the basis of the approximations which have been made (neglect of the Pauli exchange piece of the graphs, neglect of the other components of the force which builds the correlations etc...). Indeed the precision of our calculation is difficult to evaluate. This motivated a second approach, phenomenological this time, in which we use on our previous microscopic approach and which aims at the establishment of a link with $\mathrm{p}$ wave pion absorption. Why pions ? Firstly the energies involved in the dip region between the nucleon and $\Delta$ sectors, of the order of 150 to $200 \mathrm{Mev}$, are typical of low energy pions. Moreover both the magnetic photon and the $p$ wave pion have a spin isospin coupling. The difficulties are of two kinds : 


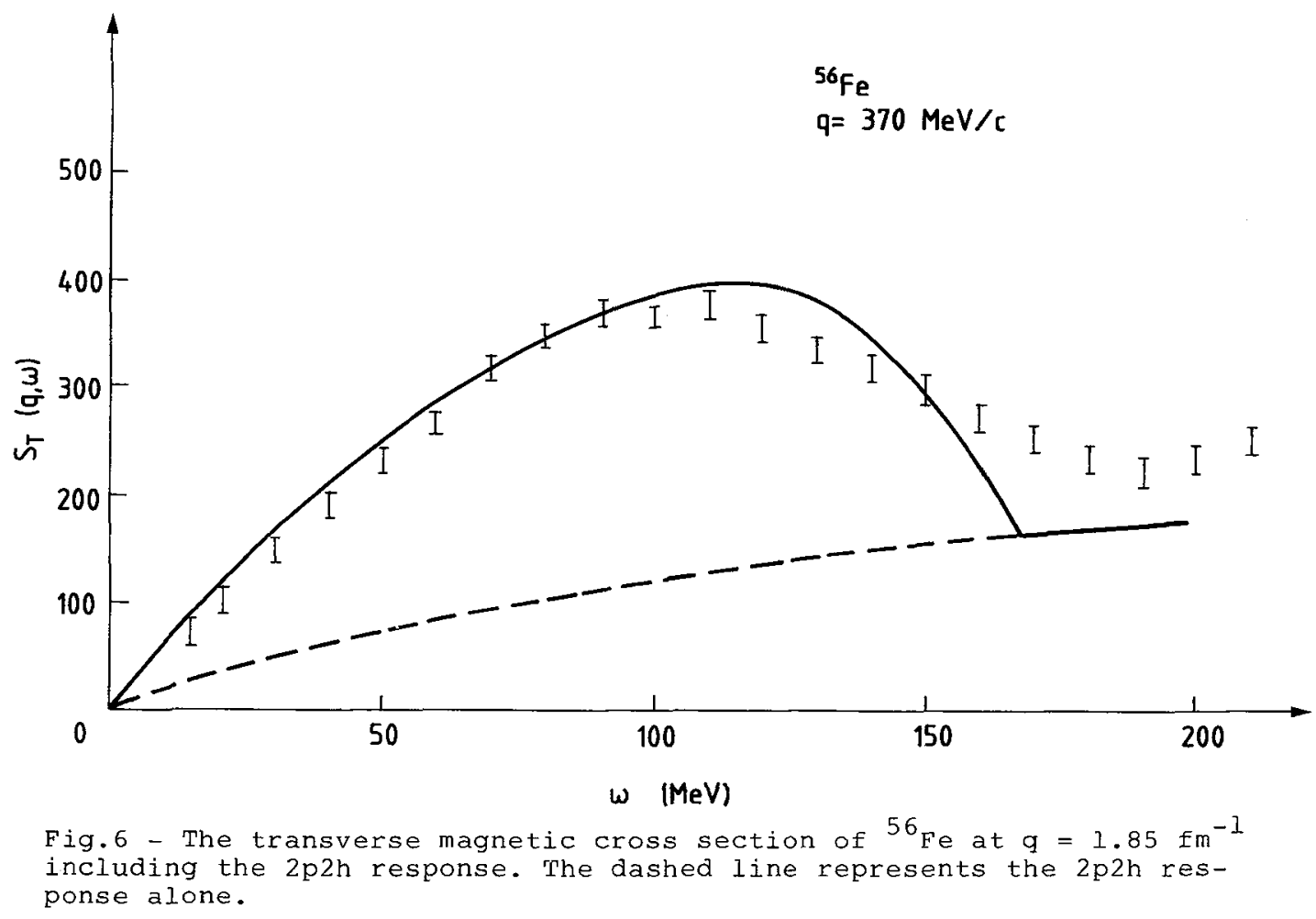

i) Since the pion and the virtual photon do not have the same mass the momenta involved in $\gamma$ and $\pi$ absorption are not the same for a given energy. In our initial and naive view this should not raise any problem as we thought of a smooth extrapolation between the two processes. In fact this is the major difficulty and it makes the link between the. two processes rather subtle.

ii) The pion has a longitudinal coupling $\vec{\sigma} . \hat{q}$ while the $\gamma$ has a transverse one $\vec{\sigma} x \hat{q}$. This difference in the nature of the coupling is at the origin of the sharp contrast between the lplh transverse and longitudinal responses and the same is expected in the $2 \mathrm{p} 2 \mathrm{~h}$ region. The way out of this difficulty has been shown in a different context by T.Ericson and Bernabeu $(6)$ with their assumption of axial locality. They showed that the link has to be made at a local level which means that it should be established between the $\gamma$ absorptive cross section and the $p$ wave $\pi$ nucleus absorptive optical potential. The reason is Eairly obvious : the pion wave is distorted while the photon is not. one has then to get rid of the distortion to link the two processes, which is realized with the introduction of the optical potential.

Our approach goes as follows. We first discuss the pion absorption process. The pion propagator is modified in the nuclear medium. In the lplh framework it becomes $D(q, w)=\left|m^{2}+q^{2}-\omega^{2}+q^{2} \tilde{\pi}(q, \omega)\right| \dot{-}^{2}$ where $q^{2} \tilde{\pi}$ is the pion self energy or optical potential with (ignoring s wave interaction)

$$
\tilde{\pi}(q, \omega)=\frac{\pi^{0}(q, \omega)}{1-g^{1} \pi^{0}(q, \omega)}
$$


In the kinematical condition of threshold pions, $q$ small and $\omega \approx m$ $x^{0}$ is purely real and totally dominated by the $\Delta$ contribution. However this description is insufficient and the $2 \mathrm{p} 2 \mathrm{~h}$ excitations add to the optical potential an absorptive piece parametrized as $i^{2} 4 \pi \operatorname{ImC}_{0} \mathrm{p}^{2}$ The value of $\mathrm{ImC}_{0}$ is accurately fixed by the $\pi$ mesic atoms data: $\operatorname{ImC}_{0}=0.005$ to $0.06 \mathrm{~m}^{-6}$. Our first and obvious idea was to test our microscopic model on this quantity. Encouraged by the success for ee' scattering we were naively confident in the success of this test. The graphs responsible for $p$ wave absorptions, as shown by Shimizu and Faessler (7), are of two kinds only

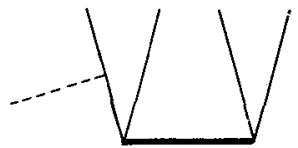

a

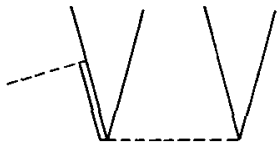

b

Fig. 7 - Graphs responsible for the absorption of p-wave pions.

The graphs of the meson exchange type (contact and pion in flight) are not relevant for $\pi$ absorption. This already puts a restriction on the link with $\gamma$ absorption which can be envisaged only inso far as these ineson exchange graphs are not dominant (which is realized in deep inelastic ee' scattering).

The result of the test was not according to expectations : our calculated value is $I \mathrm{mC}_{0} \approx 0.020 \mathrm{~m}^{-6}$, more than 3 times larger than the experimentally measured quantity. What is the reason for this discrepancy ?

Are the approximations made in the actual evaluation so crude as to fail completely on the momentum dependence? Our evaluation predicts a smooth momentum dependence which does not seem supported by the experimental data. Although we cannot exclude totally this possibility we do not think so and we attribute instead a great physical significance to this discrepancy. It is the interpretation of our model which should be questioned.

In fact there exists another parametrization of the optical potential. Quite often the absorptive piece is incorporated in the bare polarization propagator in such a way that the optical potential writes

$$
2 \omega v_{o p t}=q^{2} \frac{\underline{I}^{0}+4 \pi \zeta_{0} \rho^{2}}{I-g^{\prime}\left[\Pi^{0}+4 \pi \boldsymbol{\zeta}_{0} \rho^{2}\right]}
$$

The quantity $\boldsymbol{B}_{0}$ has both a real and an imaginary parts and Im $\boldsymbol{C}_{0}$ i) is not equal to the previous quantity $\operatorname{Im} \boldsymbol{C}_{0} \approx \operatorname{ImC}\left|1-g^{\prime}\left(\Pi^{0}+4 \pi \operatorname{Re}_{0} \mathscr{G}_{0} \rho^{2}\right)\right|^{2}$

iif is not unambiguously determined from the experimental data since it depends on $g^{\prime}$ and Re $\boldsymbol{B}_{0}$ : There are two constraints, the real and the imaginary parts of the optical potential, and three parameters, Re $\mathbb{B}_{0}$, $\operatorname{Im} \boldsymbol{b}_{0}$ and $g^{\prime}$. Tauscher $\left(8^{4}\right.$ has made a very careful analysis of the $\pi$ meslc data, trying to determine separately these 3 parameters which introduce different density dependence.

His value is $\operatorname{Im} \boldsymbol{G}_{0} \approx 0.11+0.07 \mathrm{~m}_{\pi}^{-6}$ 
larger than the previous value $\operatorname{ImC}_{0}=0.05 \mathrm{~m}_{\pi}^{-6}$. His extreme value $\operatorname{Im} \boldsymbol{b}_{0}=0.18 \mathrm{~m}_{\pi}^{-6}$ is consistent with our determination.

What is the physics behind the different parameters ? Before getting absorbed the pion can polarize the medium as represented below.

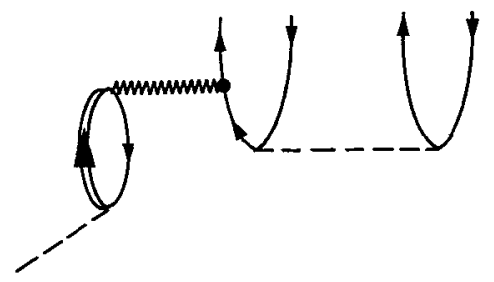

Fig.8 - Diagrams representing the vertex renormalization for the absorption of a pion in the nuclear medium.

In the last step pion exchange is not allowed because this would be a distortion effect which should be eliminated in the optical potential. but the short range interaction $g^{\prime}$ can be exchanged. This polarization process is incorporated in the quantity Imc while instead it is removed in the quantity Im $\boldsymbol{C}_{0}$ which refers to the bare graph of Fig. 7 and then it is corrected for by the RPA denominator of expression (4).

Since our microscopic model does not incorporate the vertex renormalization of Fig. 8 it should be compared to In $\mathscr{B}_{0}$ and not to $\operatorname{ImC}_{0}$, and the agreement can become much better. The condition for that is that the vertex renormalization of Fig. 8 does indeed introduce an appreciable quenching. Oset et al. (9) had previously pointed out and evaluated the importance of these vertex corrections in the real part of the p-wave optical potential (whith the graph from the $\Delta$ only). Our conclusion from the study of the $2 \mathrm{p} 2 \mathrm{~h}$ absorption of pions and photons is that there is as well a good evidence for the existence of a strong quenching in the $\mathrm{p}$ wave absorptive $\pi$ nucleus optical potential.

This result is conceptually interesting because this potential is intimately linked to the high energy tail of the G.T. strength The role of the $2 \mathrm{p} 2 \mathrm{~h}$ excitations in the low energy region ( $\mathrm{E} \leqslant 60 \mathrm{Mev}$ ) has been inyesfigated microscopically in specific cases by Berstch and Hamamoto $(10)$ and in a communication at this conference by Bortignon et al.(11) At high energy $\omega=m_{\pi}$ a nuclear matter evaluation can be obtained phenomenologically by a link with the $p$ wave absorptive potential (12).

The density of G.T. strength $G(\omega)$ at $\omega=m_{\pi}$ is proportional to ImC $_{0}$

$$
G\left(\omega=m_{\pi}\right)=\frac{m_{\pi}^{2}}{2 f^{2}} g_{A}^{2} I m C_{0} \rho A \approx 0.1 \mathrm{~A} / m_{\pi}
$$


This relation follows simply from the explicit evaluation of the $2 \mathrm{p} 2 \mathrm{~h}$ excitation by the axial current by the graphs similar to those of Fig. 7 for the pion absorption process. The axial current has a $\sigma \tau$ coupling while that of the pion is $(\sigma . q) \tau$. Removing the local pion momentum one finds the relation (5). It also follows from the more general assumption of axial locality of T.Ericson and Bernabeu (6).

Since the $p$ wave optical potential undergoes a strong quenching by the vertex renormalization of Fig. 8 we have to conclude that the G.T. strength is similarly reduced. The origin of the quenching lies for a large part in the $\Delta$ h polarization propagator but also one needs a contribution from Ree. In fact Tauscher's analysis indicates the $\mathrm{ImC}_{0}=0.18 \mathrm{~m}_{\pi}^{-6}$ corresponds to $\mathrm{g}^{\prime} \approx 0.6$ and $\operatorname{Re}_{0} \approx 0.3 \mathrm{~m}_{\pi} \mathbf{l}_{6}$.

It is interesting to draw the analogy with the situation at small $w$ values, in the region of the lplh excitations. There it is well known that a strong quenching (a factor $\approx 2$ reduction) also occurs. Part of it is attributed to the $\Delta \mathrm{h}$ polarization propagator by a mechanism similar to the one of Fig.8. In addition Desplanques (13) has drawn the attention to the possible influence of the $2 \mathrm{p} 2 \mathrm{~h}$ polarization propagator which would complete the parallel with the situation at $\omega=m_{\pi}$. The quenching of the G.T. strength seems to be a generál feature up to $\omega=m_{\pi}$.

We now come back to the link between photon and pion absorption. The same graphs of Fig. 8 which produce the quenching effect in $\pi$ absorption also apply in principle to $\gamma$ absorption. However they do not have the same effect in the last process because they are not a smooth function of the momentum. The reason is that the polarization bubble is not, as we have seen, $a_{N}$ smooth function of $q$. In the kinematical situation of $\pi$ absorption $\pi N^{2}=0$ and $\pi^{\Delta}$ is responsible for the quenching (with the possible additional influence of Re $\zeta_{0}$ ) ${ }_{N}$ while deep inelastic scattering corresponds to the region where $C_{0}^{N}$ and $\pi$ nearly cancel their effect, leaving only the influence of Re $\boldsymbol{l}_{0}$ which is presumably smaller.

One sees that the link between $\pi$ and $\gamma$ absorption is rather subtle. In order to link the two processes one has to get rid not only of the distortion of the pion but also of the vertex corrections which apply to each process but are not smooth function of the momentum. The extrapolation in $q$ then becomes smooth. The quenching correction should be applied afterwards separately for each process.

The EMC effect

We have seen that a probe with a coupling of the $\vec{\sigma} . \hat{q} \tau$ type does not interact with individual nucleons. For instance it produces a coherent chain of lplh excitations which leads to an enhancement of the lplh longitudinal response at large momenta. Also it can eject two nucleons leaving the nucleus in a $2 \mathrm{p} 2 \mathrm{~h}$ excited states, which also introduces an increase of the response over the sum of the individual nucleon values.

The spin longitudinal response has not yet been explored but these enhancement effects may have found a signature in an unexpected situation in a very high energy experiment, $\mu \mu$ ' deep inelastic scattering. This type of experiment aims at exploring the quark structure of the nucleon. 'They are similar to deep inelastic ee' scattering in nuclear physics which probes the nucleonic content of the nucleus. Simply the energies involved are much higher. The scaling variable $x$ is defined $x=Q^{2} / 2 \mathrm{~m}_{N} v$ where $v$ is the photon energy and $-Q^{2}$ its four momentum squared. The $N$ 
results are embodied in the structure functions. For practical reasons the experiments were performed on nuclei which were expected to scale the nucleon result. This turned out not to be the case. The comparison between the structure functions $F$ of $\mathrm{Fe}$ and $\mathrm{D}$ (per nucleon) revealed a difference (14). The ratio is larger than one at small $x$ values $(x<0.3)$ falling below one beyond (14-15). This is the EMC effect. The pionic interpretation of this effect concerns the small $x$ enhancement (although a complete description should treat simultaneously all $x$ values). At small $x$ the contribution of the sea quarks (qq pairs) is dominant; the idea is that for the nucleon part of this sea is in the form of pions, (16) that there is an increase of the number of pions in the nucleus (17) and that this increase is linked to the previous considerations on the enhancement of the response to a pionic type of probe $(8)$.

I cannot enter here in the details but with reasonable values of the parameters (in particular the Landau Migdal parameters g') we find an effect of the observed magnitude. As expected we are quite sensitive to the input parameters, in particular to g'. The contribution arising from the lplh enhancement is shown in Fig.9. We have also explored in a preliminary way the role of the $2 \mathrm{p} 2 \mathrm{~h}$ excitations and we have found it appreciable.

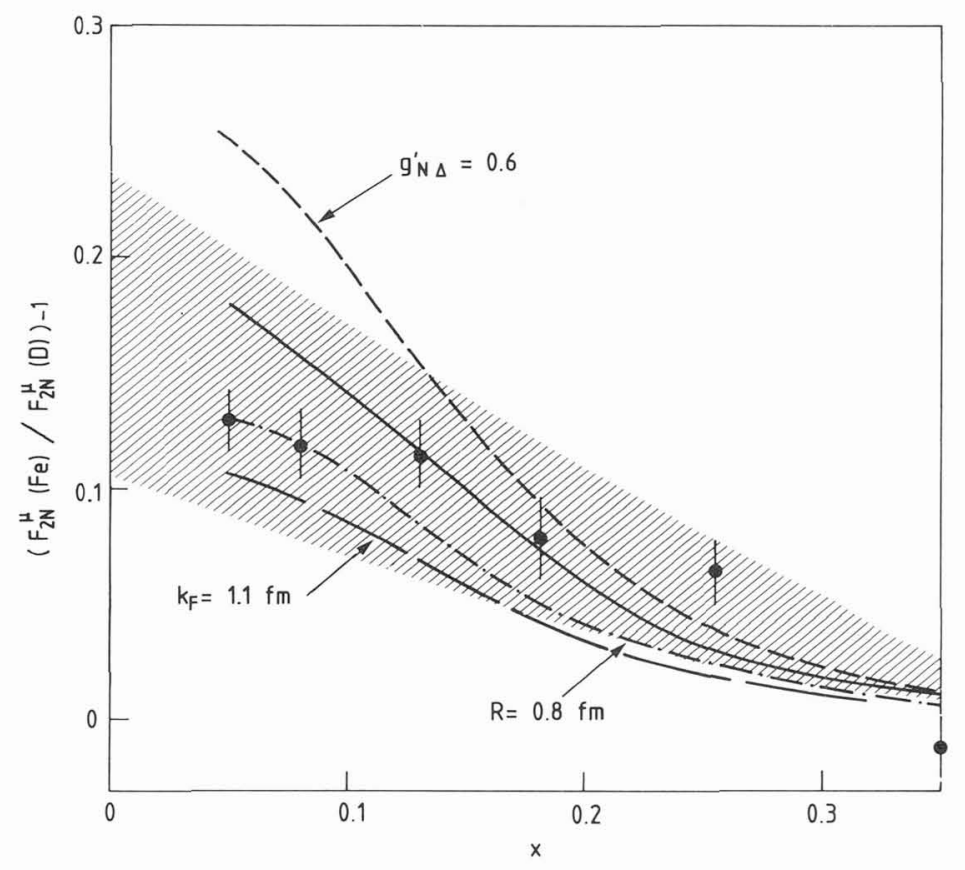

Fig.9 - The fractional increase in the ratio of the structure function in $\mathrm{Fe}$ compared with $\mathrm{D}$, as a function of $\mathrm{x}\left(=\mathrm{Q}^{2} / 2 \mathrm{~m}_{\mathrm{N}} \nu\right)$. The data are from the EMC collaboration, and the shaded area indicates possible systematic errors. The standard input (solid curve) for $F e$ is $k_{F}=1.30$ $\mathrm{fm}^{-2}, \mathrm{~g}^{\prime} \mathrm{NN}=\mathrm{g}^{\prime} \mathrm{N \Delta}=\mathrm{g}^{\prime} \Delta \mathrm{N}^{\prime}=0.7, \mathrm{a} \mathrm{bag}$ radius of $0.7 \mathrm{fm}$ in $\mathrm{F}\left(\mathrm{q}^{2}\right)$, and $\Gamma\left(q^{2}\right)$ is a dipole of ${ }^{\Delta}$ mass $1.67 \mathrm{GeV}$. In the other curves the effect of altering any single one of these parameters is shown. 
The existence of an enhancement of (fyp pion content of the nucleus has been confirmed by Friman et al who give for the excess $\delta n_{\pi} / A$ the following numbers

$\begin{array}{lc}{ }^{4} \mathrm{He} & 0.09 \\ 27_{\mathrm{Al}} / \mathrm{A} \\ { }^{56} \mathrm{Fe} & 0.11 \\ { }^{208} \mathrm{~Pb} & 0.12 \\ \end{array}$

It is both surprising and gratifying that intermediate energy physics has a chance to have a relevance for high energy experiments.

\section{Acknowledgements}

These notes would not exist without the people with whom I shared the excitement of the work, in particular W.Alberico, J.Delorme, A.Molinari and A.Thomas. I thank Dr.Meziani for communicating his results.

\section{References}

1) ALBERICO W.M, ERICSON M. and MOLINARI A., Nucl.Phys. A 386 (1982) 412.

2) Private communication Erom J.MOSS.

3) MCCARTHY J.S., Nucl. Phys., A 335, (1980) 27. ALTEMUS R.M, Thesis, Dept. of Physics, University of Virginia (1980)

4) Private communication from MEZIANI.

5) ALBERICO W, ERICSON M. and MOLINARI A., CERN preprint Th3635, 1983 to appear in Annals of Phys.

6) ERICSON T.E.O. and BERNABEU J., Phys.Lett. $70 \mathrm{~B}$ (1977) 170.

7) SHIMIZU K. and FAESSLER A., Nucl.Phys., A 333, (1980) 495

8) TAUSCHER L., private communication.

9) OSET E. and WEISE W., Nucl.Phys. A 319 (1979) 477 ; OSET E. and W.WEISE, Nucl.PhyS. A 329 (1979) 365;

OSET E., WEISE $W$ and BROCKMANN R. Phys. Lett. 82 B (1979) 344

10) BERTSCH' G.F. and I.HAMAMOTO, Phys. Rev. 26C (1982) 1323

11) BORTIGNON P.F, BROGLIA R.A. and XIA KE DING communication 7.3 at this conference.

12) ERICSON M. Phys. Lett. 120 B (1983), 285.

13) DESPLANQUES B., 7e Session d'Etudes Biennale de Physique Nucléaire Aussois 14-18 Mars 1983, Lycen 8302, S.6. and communication 7.5 at this conference.

1.4) AUBERT J.J. and co-workers, Phys.Lett., 123B,(1983) 275

15) BODEK A. and co-workers, Phys.Rev.Lett., 50, (1983) 1431

1.6) SULLIVAN J.D., Phys.Rev., D5, (1972) 1732

17) LLEWELLYN SMITH C.H., Phys. Lett 128 B, (1983) 107

18) ERICSON M. and THOMAS A.W., Phys.Lett. 128B,(1983) 112

19) FRIMAN B.L, PANDHARIPANDE V.R. and WIRINGA R.B., preprint ILL-(Nu) 83-15, to appear in Phys.Rev. Lett. 